\title{
Effects of Magnetic Field on Biological Systems
}

\author{
Masateru IKEHATA, Ph.D. \\ Tamami KAWASAKI, Ph.D. \\ Senior Researcher, \\ Assistant Senior Researcher, \\ Biotechnology Laboratory, Environmental Engineering Division
}

\author{
Yuji SUZUKI, Ph.D. \\ Associate Professor, \\ Hidesuke SHIMIZU, Ph.D., M.D. \\ Professor,
}

Jikei University School of Medicine

\author{
Takao KOANA, Ph.D. \\ Senior Visiting Researcher, \\ Low Dose Radiation Center, Central Research Institute for Electric Power Industry
}

\begin{abstract}
We investigated the biological effects of the magnetic field produced in the superconducting Maglev system environment, such as the extremely low frequency intermittent pulsed magnetic field (LFIP-MF) that is generated along the guideway when a Maglev train passes. We observed no evidence of any biological effects from exposure to the Maglev system's LFIP-MF in "in vivo" long-term exposure experiments using AKR/JSea mice, in "in vitro" short-term exposure experiments as bacterial mutation assay, a yeast mutation assay or an analysis of global gene expression profiles in yeast. These results suggest that the Maglev system's environmental LFIP-MF does not have any health / biological effects that would need to be considered as part of an environmental assessment.
\end{abstract}

Keywords: electric and magnetic field, safety, mutagenicity, gene expression, Maglev

\section{Introduction}

The superconducting magnetically levitated (Maglev) train uses electromagnetic force for propulsion, levitation and guidance. To produce the electromagnetic force, the system uses a ground coil on the track and an onboard superconducting magnet (SCM). Because of the SCM used on the train and its movement, the magnetic field that is generated along the trackside environment has unique features, such as the extremely low frequency intermittent pulsed magnetic field (LFIP-MF). Information on the safety of this LFIP-MF had not yet been determined, therefore a safety assessment was necessary. Previously our studies had showed several biological effects from strong single-component magnetic fields such as static or $50 \mathrm{~Hz}$ time-varying magnetic fields $\mathrm{s}^{1,2)}$. These findings and an abundance of other evidence led us to increase our understanding of the nature of the biological effects of magnetic fields. However, Maglev development is now reaching maturity, so a safety assessment of a realistic model of its environmental magnetic field is required for its practical use.

This study aimed to estimate the biological effects of the magnetic field in the area around the Maglev track and contribute to an assessment of the system's health risk. In this report, we focused on the magnetic field associated with the SCM moving within its environment and conducted a mouse survival study to determine the effects on leukemia progression from long-term exposure, a bacterial mutation assay and a yeast mutation assay for short-term exposure. Moreover, a gene expression profile was analyzed in yeast.
2. Development of exposure devices for generating simulated magnetic field in Maglev environment

\subsection{Magnetic field in Maglev environment and exposure condition}

An environmental magnetic field at the same height and $7 \mathrm{~m}$ away from the train was measured by Sasakawa et $\mathrm{al}^{3)}$. The magnetic field waveform was synthesized using this data and assumed for a commercial train set of 16 cars with 17 bogies. Moreover, the maximum magnetic flux density was $2.7 \mathrm{mT}$ (Tesla; $1 \mathrm{~T}=10,000 \mathrm{Gauss}$ ) that is approximately 15 times higher than the environmental level used to estimate the most stringent exposure conditions. LFIP-MF was applied every 30 seconds, 10 times more frequently than was assumed for long-term exposure to the commercial operation, and every $60 \mathrm{sec}-$ onds for short-term exposure experiments.

\subsection{Development of exposure devices}

Two types of exposure devices were developed to realize the above exposure conditions. One device was for in vitro short-term exposure, consisting of two horizontal, orthogonal-oriented Merritt shape coils ${ }^{4)}$. Each Merritt shape coil has four subcoils to make up one axis. The exposure space was $15 \times 15 \times 18 \mathrm{~cm}$ with a magnetic flux density fluctuation of approximately $10 \%$. To minimize the artifact, two exposure coils were made and used for exposure or sham exposure. Fig. 1 shows pictures of the exposure device, which was driven by an originally developed control unit (MASM-3Dmag-10A, Jyujyo Denshi Co.) and used to synthesize two-dimensional wave- 


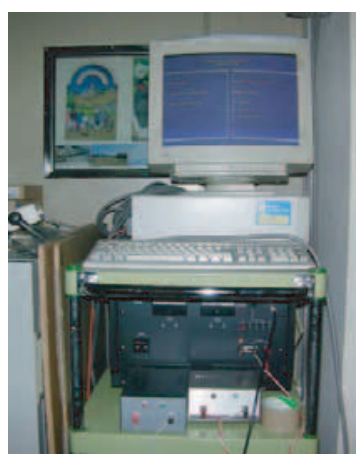

Control unit

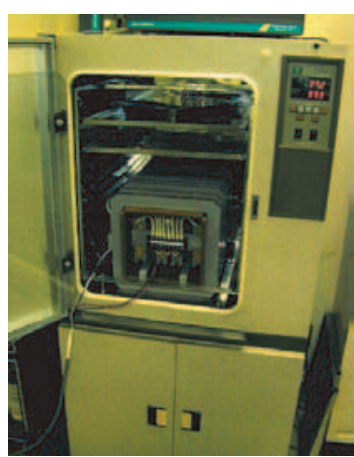

Exposure coil in incubator
Fig. 1 Exposure device and control unit for in vitro experiment

forms each waveform being inputted into a bipolar power amplifier (BWS-40-15, Takasago Co.) to amplify the wave and provide power to the exposure coils, respectively. The LFIP-MF waveform used in this study is described in 2.1 and shown in Fig. 2.

The other exposure device was for in vivo long-term exposure and had three Merritt coils for three-dimensional reconstruction of the environment's magnetic field. A control unit identical to that used for the in vivo sys-

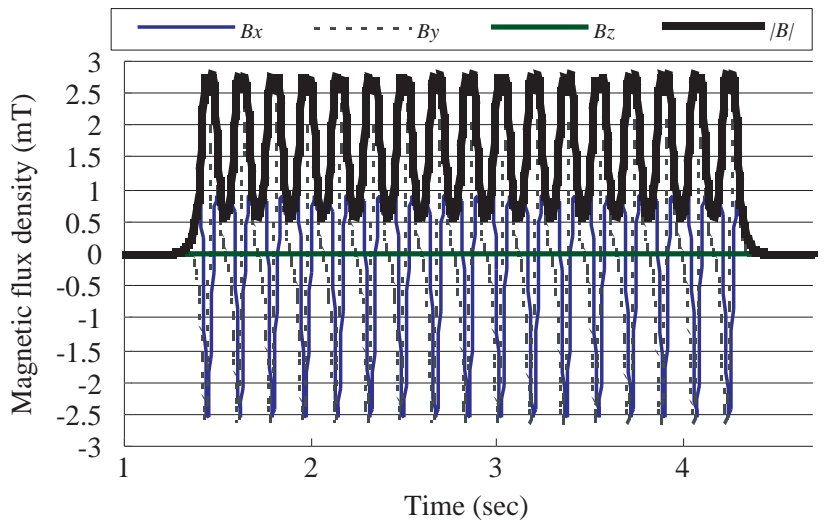

Fig. 2 LFIP-MF waveform used in this study This waveform simulated 16 cars with 17 bogies at $500 \mathrm{~km} / \mathrm{h}$ and a magnetic flux density approximately 15 times higher than actual data.

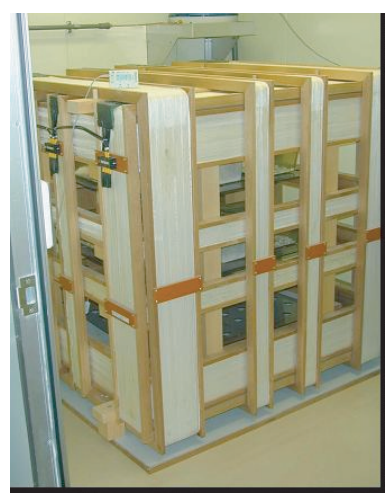

Exposure coil No.1

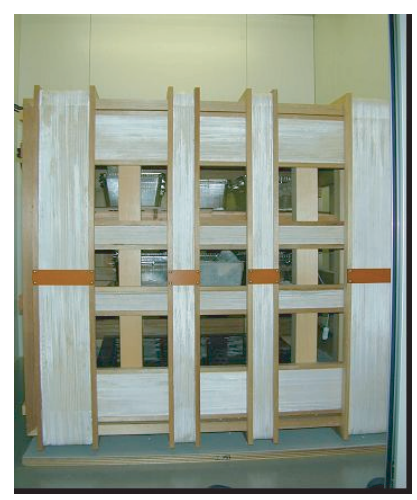

Exposure coil No.2
Fig. 3 Exposure devices for in vivo experiment tem drove each Merritt coil. The exposure space was 150 $\times 150 \times 150 \mathrm{~cm}$ and 15 animal cages (each capable of holding up to 10 mice) could be exposed to the magnetic field with $10 \%$ fluctuation. Two exposure coils were made, located in a room with constant temperature and humidity and used for exposure or sham exposure experiments respectively to avoid subjecting the test animals to any physiological stress (Fig. 3).

\section{Effects of long-term exposure on progression of lymphatic leukemia in AKR/JSea mice}

Our previous data ${ }^{1)}$ ) show the mutagenicity and comutagenicity from static and time-varying magnetic fields, respectively. These results suggested that exposure to magnetic fields in the environment carries an extremely small risk to our health compared with other environmental toxicants. On the other hand, epidemiological studies show the existence of a weak association between child leukemia and exposure to magnetic fields near power lines ${ }^{5)}$. In this chapter, to examine the effects of magnetic fields on leukemia progression, we used AKR/JSea mice that develop lymphatic leukemia, which has become the main cause of death in mice older than 25 weeks.

\subsection{Materials and methods}

AKR/JSea mice (Kyudo Co. Ltd., Japan) were used as leukemia progression models. In total, 105 male and 105 female mice were used in the first experiment and 105 female mice were used in subsequent experiments. The mice were purchased from the breeder at 5 weeks of age and randomly divided 10 mice per cage. The mice had access to food (CRF-1, Oriental Yeast Co., Japan) and water ad libitum. After three weeks of acclimation and quarantine, the mice (now 8 weeks old) were randomly divided into two groups (5 cages/group) and each placed in the two exposure coils. A control unit energized one coil as an exposure group while the other was not energized to act as a sham exposure group. Under these exposure conditions, mice in the exposure group were exposed to LFIP-MF every 30 seconds, except during care time, throughout their lives. No significant vibration, sound or temperature rise was observed within the exposure space during the exposure. A temperature of $22 \pm 1$ ${ }^{\circ} \mathrm{C}$ and $50 \pm 10 \%$ humidity was maintained in both the exposure room and the sham exposure room. Light was provided in a $12 \mathrm{~h}$ light/12h dark cycle. The mice were checked twice a week by palpation and by recording their weight. Because the main cause of death among these mice is leukemia, the progression of which is revealed by such typical symptoms as raised hair, lack of movement, weight loss, thymus hyperplasia one or two weeks before death, the survival curve in both groups was recorded and compared as an index of leukemia progression. This experiment was repeated twice and in addition to the replication, two sham exposure experiments carried out to confirm the spontaneous life span of AKR/JSea mice in this experimental facility with female mice only. The result was analyzed using log-rank and generalized Wilcoxon tests. 


\subsection{Results}

Mice started to die from 25 weeks of age and half of them were dead by 35 weeks in all of the experiments. The survival curves were similar for both exposure and sham exposure groups in each experiment, with the longest survivor varying from 74 weeks to 112 weeks. This difference would result in an uncontrollable factor in a breeding colony. Mice showed moribund symptoms such as raised hair, lack of movement, weight loss, thymus hyperplasia one or two weeks before death. Autopsies carried out on several mice showed thymus hyperplasia, spleen hypertrophy and other typical symptoms associated with lymphatic leukemia. No other causes of death were observed in this study. Fig. 4 shows the survival rate time line from the first experiment, in which a slightly higher death rate was observed among exposed female mice between 35 and 45 weeks of age. However, statistical analysis showed no difference $(\mathrm{p}=0.89$ in the log-rank test, $\mathrm{p}=0.49$ in the generalized Wilcoxon test). On the other hand, the survival time line was almost identical in male mice (data not shown). These data suggest that exposure to LFIP-MF in the Maglev environment would have a possible effect on the progression of lymphatic leukemia in female AKR/JSea mice because of the tendency toward an increase in death rate at 35-45 weeks. However, the survival rate time line from other sham experiments (Experiments 2 and 3 ) fell between the exposure and sham exposure groups of the first experiment (Fig.4). This result suggests that the observed results (the effective tendency in exposure group) from the first experiment were caused by an unknown artifact during the experiment. However, because of the progressive tendency in the exposure group in first experiment, we decided to perform a replication study. The result showed not only the absence of the effect but also no tendency toward an increase in death rate in the survival rate time line (Fig. 5). Statistical analysis again showed no significant difference between the exposure and the sham exposure group $(p=0.23$ in the log-rank test, $p=0.46$ in the generalized

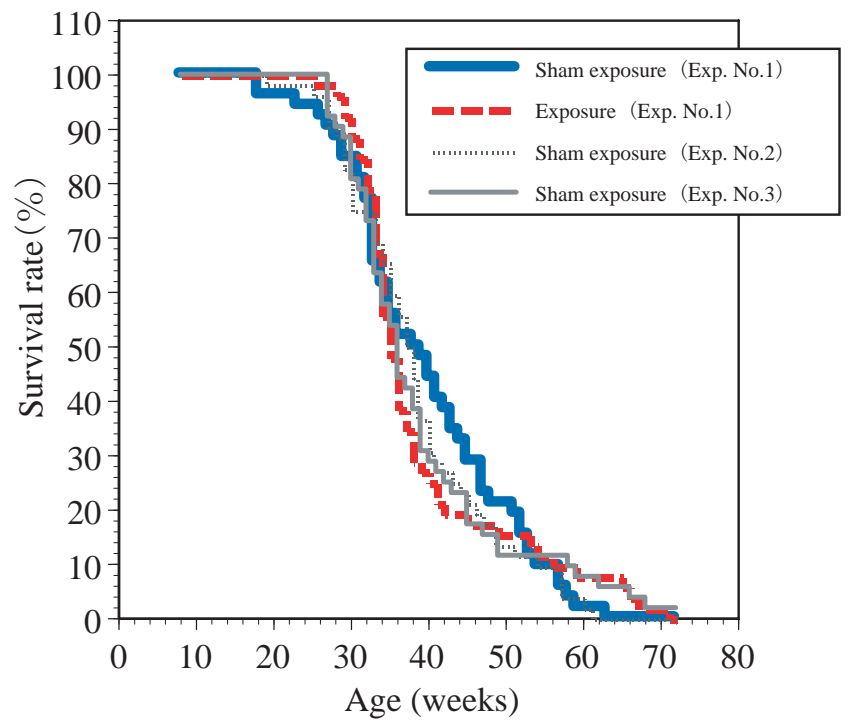

Fig. 4 Survival curves of female AKR/JSea mice in longterm exposure experiment

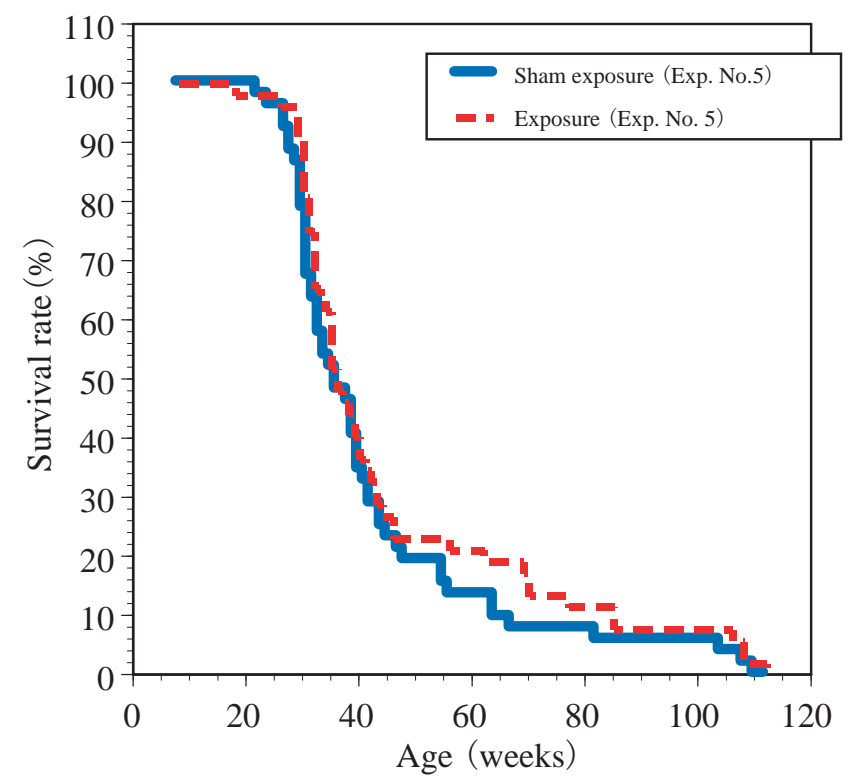

Fig. 5 Survival curves in replication study

Wilcoxon test). These results show that LFIP-MF in the Maglev environment does not affect the progression of lymphatic leukemia in AKR/JSea mice. This evaluation also suggests that a real environmental magnetic field 1/ 15 in magnetic flux density and 1/10 in frequency would not have any effect on the progression of lymphatic leukemia in these mice.

\section{Cellular-level biological effects from short-term exposure}

In vivo study experiments provide direct and essential results for estimating the risk from magnetic fields, however, it is important to apply various indices to estimate safety. Though there are many approaches to estimate biological effects, mutation assays in bacteria and yeast were employed to provide in vitro genotoxic study, a gene expression analysis using cDNA microarray technique in yeast performed to explore the cellular response in vitro.

\subsection{Bacterial mutation assay}

The bacterial mutation assay is one of the most conventional screening tests for genotoxicity and is included in many guidelines and test batteries such as the OECD Guidelines for the Testing of Chemicals ${ }^{6}$. This test is able to estimate genotoxicity/carcinogenicity qualitatively and, in addition, data for a large number of industrial and environmental chemical substances covered in this assay are available. It is important to refer to these data to estimate and understand the risk of exposure to magnetic fields.

Two routinely used tester strains, S. typhimurium TA98 and S. typhimurium TA100, were used in this study. These strains have a histidine biosynthesis deficiency caused by the mutation of one responsible gene. Therefore, if they are poured onto agar plate media without histidine, they cannot grow. However, if mutagenesis has occurred on the 
responsible gene and the mutation-causing function is restored, the cell starts growing and a colony will appear on the plate. This reverse mutation is the test index. These colonies are counted and used for the analysis of the mutation frequency.

LFIP-MF exposure conditions were the same as Chapter 2 except in the exposure interval, which was 60 seconds (30 seconds in Chapter 2). Frozen test bacteria stock was thawed and incubated overnight in nutrient broth at $37{ }^{\circ} \mathrm{C}$ with reciprocal shaking. After incubation, the cells were poured on to minimal glucose agar plates $(\phi 90 \mathrm{~mm})$ without histidine. These plates were randomly divided into two groups and one group placed in the exposure coil and exposed to LFIP-MF for $48 \mathrm{hr}$ at $37^{\circ} \mathrm{C}$ while the other was placed in another exposure coil and incubated at the same temperature for the same duration without LFIP-MF. After the exposure period, the bacterial colonies were counted and the mutation frequency was analyzed.

As a result, we observed no significant difference in mutation frequency between the exposure and sham exposure group, while positive control (treatment with known chemical mutagen AF-2) showed a large increase in mutation frequency (Table 1). This result suggests that simulated LFIP-MF in the Maglev environment does not have any mutagenic potential or might have weak mutagenic potential that is too small to detect.

Table 1 Result of bacterial mutation assay in LFIP-MF

\begin{tabular}{lccc}
\hline & S9mix $^{\mathrm{a}}$ & \multicolumn{3}{c}{ HIS mutant per $10^{8}$ cells } \\
\cline { 3 - 4 } & & S. typhimurium TA98 & S. typhimuriumu $\mathrm{TA100}$ \\
\hline Control & - & $37 \pm 7.8^{\mathrm{b}}$ & $101 \pm 6.6$ \\
Exposure & - & $36 \pm 7.4$ & $110 \pm 12.2$ \\
AF-2 $^{\mathrm{c}}$ & - & $360 \pm 34.9$ & $494 \pm 31.4$ \\
& & & \\
Control & + & $27 \pm 2.4$ & $108 \pm 8.6$ \\
Exposure & + & $31 \pm 8.4$ & $99 \pm 6.8$ \\
2-Aminoanthrathene & + & $1441 \pm 37.9$ & $653 \pm 5.2$
\end{tabular}

a, Rat liver homogenate for metabolic activation

b, Standard deviation

c, 2-(2-furyl)-3-(5-nitro-2-furyl)acrylamide (CAS No. 3688-53-7)

\subsection{Yeast mutation assay}

Although the bacterial mutation assay is a sensitive, simple and accurate test to examine mutagenicity, there are several types of mutations and mutagenesis mechanisms that it cannot detect as they depend on differences in cellular physiology.

For example, prokaryote bacteria such as E. coli do not have nuclei and have one copy of their own genome tangled in cells called nucleoids. On the other hand, eukaryote cells like fungi, rodents and humans have two sets of genomes (e.g. paternal and maternal genomes in humans) which means double the basic chromosome number condensed in chromosomes in nuclei. This difference causes different types of mutagenesis in each organism. In bacteria, point mutations, frame shifts or small deletions are major mutations. In addition to these types of mutations, large deletions, loss of heterozygote, gene conversions and chromosome recombinations occur fre- quently in eukaryote cells. It is therefore important to examine various types of mutations to evaluate genotoxic effects as a risk. For this reason, we used budding yeast Saccharomyces cerevisiae XD83 to detect multiple genetic endpoints ${ }^{7}$. With this strain, we can simultaneously detect various genetic endpoints (point mutations such as frame shifts and base substitutions, gene conversion/recombination, deletion and mitotic crossover) in the same genetic background and under the same experimental conditions. In this study, we focused on base substitution and gene conversion/recombination. In this strain, $A R G 4$ allele includes a heterozygous mutation ( $\arg 4-4$ on one chromosome and $\arg 4-17$ on another chromosome) and it is known that reversion of $A R G 4$ allele is caused by gene recombination or conversion. On the other hand, lys 1-1 ochre mutation is homozygous. It is known that reversion of LYS1 allele is caused by point mutation of lys 1-1 on either chromosome.

To detect the point mutation frequency on lys 1-1, cells were poured onto low-lysine synthetic complete plates. To detect the gene conversion frequency on $A R G 4$ allele (between $\arg 4-4$ and $\arg 4-17$ ), cells were poured onto lowarginine synthetic complete plates. At least six plates were made for each condition and randomly divided into two groups. One group was exposed to LFIP-MF for 5 days at $35 \pm 1{ }^{\circ} \mathrm{C}$. The other group was incubated as a sham control. The number of colonies on each plate was scored as reverse mutant and the mutation frequency calculated.

Table 2 shows the result of this experiment in this study and other previous results (5T static magnetic field and $50 \mathrm{~Hz}, 40 \mathrm{mT}$ magnetic field). From this result, no significant difference in mutation frequency was observed in either point mutation or gene conversion/recombination by exposure to LFIP-MF in the Maglev environment. Under other exposure conditions, a $50 \mathrm{~Hz}, 40 \mathrm{mT}$ magnetic field also revealed no mutagenic activity in either mutation index. In contrast, a slight but significant increase in gene conversion/recombination was observed by exposure to a $5 \mathrm{~T}$ static magnetic field. Moreover, ultraviolet (UVC) irradiation, which was estimated to be approximately $1 / 5$ to $1 / 10$ that of the average sunlight in Japan as a positive control, caused a significant increase in both ARG4 and LYS1 reverse mutations (Table 2). The positive control result suggests that this assay has enough sensitivity to the mutagenic agent. These data suggest that the extent of mutagenicity of MFs was estimated to be

\section{Table 2 Result of the yeast mutation assay for various magnetic fields in S. cerevisiae XD83}

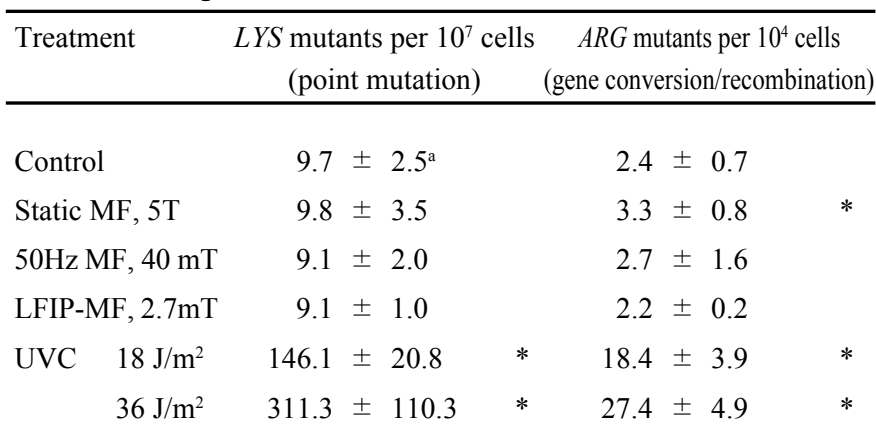

*Significantly higher than the control group at $1 \%$ level 
extremely small even in a $5 \mathrm{~T}$ static magnetic field, which is at least 10,000 times stronger than those in the environment, since weak UVC exposure $\left(18 \mathrm{~J} / \mathrm{m}^{2}\right)$ is approximately 20 times more effective than a $5 \mathrm{~T}$ static magnetic field.

\subsection{Gene expression profile in yeast}

Recent findings from cancer research show the existence of non-mutagenic carcinogens and so the carcinogenesis process must be including gene mutation and other factors. To estimate non-mutagenic carcinogenesis, an analysis of gene expression profiles is an important approach. There are several high-throughput methods to analyze whole genome expression profiles, such as cDNA microarray, oligo-DNA microarray, the serial analysis of gene expression (SAGE) and Hi-CEP. These methods are able to analyze simultaneously the expression of several tens of thousands of genes. In this study, the cDNA microarray method was employed to determine the effect of LFIP-MF in the Maglev environment on the gene expression profile in yeast Saccharomyces cerevisiae cells.

A yeast chip that includes $5,876 \mathrm{cDNA}$ out of $6,400 \mathrm{~S}$. cerevisiae ORFs (DNA Chip Research Inc., Japan,) was used. S. cerevisiae 4388 (wild-type strain) was used in this experiment. The cells were incubated overnight in yeast extract, peptone and dextrose (YPD) medium and $1 / 1000$ by volume of the cell suspension was resuspended to provide $25 \mathrm{ml}$ of fresh YPD medium in a $25 \mathrm{ml}$ media bottle. The culture bottle was capped tightly and incubated with/without LFIP-MF at $35{ }^{\circ} \mathrm{C}$ for $24 \mathrm{hrs}$. After exposure, the cells were harvested by centrifugation and the mRNA was extracted. mRNA isolation, cDNA preparation and hybridization were performed following the protocols provided by the manufacturer, with only slight modification.

The data obtained from each spot were normalized and the ratio of gene expression in exposed cells compared with that in control cells calculated. Clustering analysis was performed with selected genes related to several cellular processes (TCA cycle, DNA damage response, oxidative stress response, protein synthesis and other stress responses which were annotated by the Saccharomyces genome database (http://genome-www.stanford.edu/ Saccharomyces/)) using Cluster and Tree-View software (Stanford Univ., USA). At least two independent experiments were performed under every experimental condition.

The results showed no significant differences in gene expression profiles between the exposure and sham exposure groups for LFIP-MF in the Maglev environment. Fig. 6 shows the change in expression of TCA cycle-related genes under various treatments including LFIP-MF in the Maglev environment and other previous experiments (5T static magnetic field, $50 \mathrm{~Hz}, 40 \mathrm{mT}$ magnetic field and oxidative stress). The TCA cycle-related genes were selected as candidates because exposure to a $14 \mathrm{~T}$ static magnetic field caused the induction of several genes in this biological pathway in our previous study ${ }^{8)}$. In this figure, the ordinate axis indicates the ratio of exposure to sham exposure in the gene expression of individual genes. The data shows that oxidative stress caused significant in-

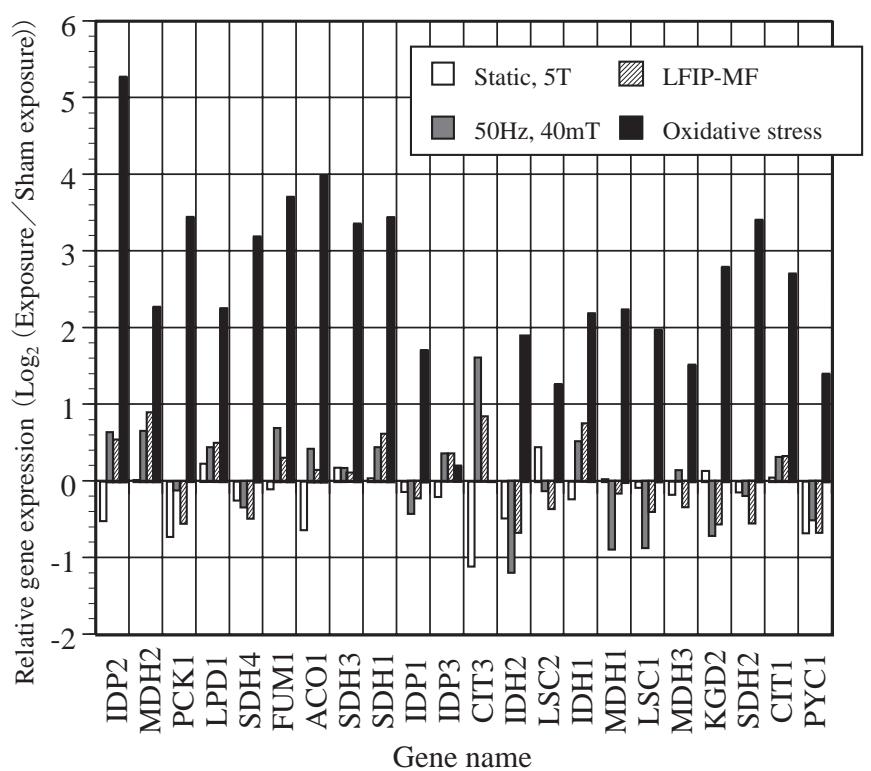

\section{Fig. 6 Gene expression profile of TCA cycle-related genes in $S$. cerevisal by exposure to various magnetic fields}

duction of these genes while other treatments did not affect even those exposed to the $5 \mathrm{~T}$ static or $50 \mathrm{~Hz}, 40 \mathrm{mT}$ magnetic fields. These results suggest LFIP-MF in Maglev environment does not have an effect on gene expression in yeast cells or, even if the effect exists, the effect is too small to consider its risk among other risk factors in the environment.

\section{Conclusion}

In this study, in vivo long-term exposure experiments using proleukemic AKR/JSea mice were carried out to evaluate the effect of LFIP-MF in the Maglev environment on leukemia progression and in vitro short-term exposure using bacteria and yeast to evaluate its effect on genotoxicity and gene expression. From the results, no significant effect from exposure to LFIP-MF in the Maglev environment was observed in any of the experiments we employed. These suggest that LFIP-MF in the Maglev environment has no biological effect, or its biological effects would be so small as to be undetectable by generally accepted safety evaluation methods. Therefore, the risk from LFIP-MF in the Maglev environment, especially that related to genotoxicity, is estimated to be negligible in the practical sense. In further studies, the biological effect of LFIP-MF in the Maglev car and its effects on the function of differentiated cells should be studied to establish further experimental evidence of its effects. In addition, effects on medical implants should be studied.

On the other hand, EU directive 2004/40/EC on the minimum health and safety requirements regarding the exposure of workers to the risks arising from physical agents (electromagnetic fields) has been approved ${ }^{9}$. This directive requires compliance with International Commission for Non-Ionizing Radiation Protection (ICNIRP) guidelines for limiting exposure to electric, magnetic and 
electromagnetic fields ${ }^{10,11)}$ and establishes the risk assessment procedure for its compliance. The member states are required to bring into force the laws, regulations and administrative provisions necessary to comply with this directive by April 2008. Moreover, the World Health Organization and supporting international organizations such as ICNIRP and the International Agency for Research on Cancer (IARC) have been reviewing recent scientific findings with regard to the biological effects or health effects of electromagnetic fields ${ }^{12}$. A series of environmental health criteria will be published within a few years. It will be important to keep pace with these trends.

Finally, we should provide a comprehensive report on Maglev system safety for the Japanese public ahead of any practical application. For this purpose, an evaluation of dosimetry such as the density of the current induced within the human body by LFIP-MF in the Maglev environment will provide clues to establish a comprehensive outcome that incorporates biological studies.

\section{References}

1) Ikehata, M., Koana, T., Suzuki, Y., Shimizu, H., Nakagawa, M., "Mutagenicity and co-mutagenicity of static magnetic fields detected by bacterial mutation assay," Mut. Res., Vol. 427, pp. 147-156, 1999

2) Koana, T., Okada, M.O., Takashima, Y., Ikehata, M. and Miyakoshi, J., "Involvement of eddy currents in the mutagenicity of ELF magnetic fields," Mut. Res., Vol. 476, pp. 55-62, 2001

3) Sasakawa, T., Ikehata, M. and Kitano, J., "Environmental Magnetic Fields in the Yamanashi Test Line,"
RTRI QR, Vol. 39, pp. 78-81, 1998

4) Merritt, R., Purcell, C. and Stroink, G. "Uniform Magnetic Field Produced by Three, Four, Five Square Coils," Rev. Sci. Instrum. Vol. 54, No. 7, pp. 879-882, 1983

5) Ahlbom, I.C., Cardis, E., Green, A., Linet, M., Savitz, D. and Swerdlow, A., ICNIRP (International Commission for Non-Ionizing Radiation Protection) Standing Committee on Epidemiology, "Review of the epidemiologic literature on EMF and Health, "Environ Health Perspect. Vol. 109, Suppl. 6, pp. 911-33, 2001

6) OECD Guidelines for the Testing of Chemicals / Section 4: Health Effects Test No. 471: Bacterial Reverse Mutation Test, 1997

7) Dixon, M.L. and Mortimer, R.K., "A yeast screening system for simultaneously monitoring multiple genetic endpoints," Mut. Res. Vol. 161, pp. 49-64, 1986

8) Ikehata, M., Iwasaka, M., Miyakoshi, J., Ueno S., and Koana, T., "Effects of intense magnetic fields on sedimentation pattern and gene expression profile in budding yeast," J. Appl. Phys., Vol. 93, pp. 6724-26, 2003

9) European Union, Directive 2004/40/EC the minimum health and safety requirements regarding the exposure of workers to the risks arising from physical agents (electromagnetic fields), Official Journal of European Union, L184, 1-9, 2004

10) ICNIRP, Guidelines on limits of exposure to static magnetic fields. Health Phys., 66, pp. 100-106, 1994

11) ICNIRP, Guidelines for limiting exposure to timevarying electric, magnetic and electromagnetic fields (up to $300 \mathrm{GHz}$ ). Health Phys., 74, pp. 494-522, 1998

12) World Health Organization, The International EMF Project, http://www.who.int/peh-emf/project/en/ 